\title{
Effet D'une Sollicitation En Face A Face Et D'un Contact Tactile Sur L'acception Du Don Du Sang : Une Evaluation Lors D'une Collecte En Milieu Universitaire
}

\author{
Nicolas Guéguen, Professor \\ Virginie Sire, Assistant-professor \\ University of Bretagne-Sud, France
}

doi: 10.19044/esj.2016.v12n2p51 URL:http://dx.doi.org/10.19044/esj.2016.v12n2p51

\begin{abstract}
The positive effect of touching on compliance with a request has been widely reported in the social psychology literature. However its effect on blood donation has never been tested. Yet, research found that many further compliance gaining procedures were not efficient with blood donation requests. University students were solicited to give blood during a special one-day drive. Solicitations were made through face-to-face interactions. During the solicitation some participants were lightly touched or not on the forearm. No difference was found between the two experimental groups in the number of participants who agreed to give blood. Such results show that tactile contact is not an effective technique for increasing compliance with a blood donation request, and confirm previous studies which found that other compliance-growth techniques are not effective with blood donation requests. However, if was found that, compared to previous blood campaigns conducted in the same setting, more blood donations were observed attesting that face to face solicitation of potential blood donors significantly increased donations.
\end{abstract}

Keywords: Tactile contact, Compliance, Blood Donation

\section{Résumé}

Les effets positifs du toucher sur l'acceptation de requêtes ont été mis en évidence à de nombreuses reprises dans la littérature psychosociale. Cependant, son efficacité dans le cadre du don du sang n'a jamais été testée. En outre, on a observé que de nombreuses techniques d'influence s'avèrent non efficientes dans le cadre du don du sang. Des étudiants ont été sollicités pour faire un don dans le cadre d'une campagne de collecte sur site. Les 
sollicitations étaient faites en face-à-face. Pendant cette interaction, des participants étaient légèrement touchés sur l'avant-bras ou pas. Aucune différence ne sera trouvée entre les groupes expérimentaux en ce qui concerne le nombre de participants ayant accepté de donner leur sang. De tels résultats montrent que la technique du contact tactile ne s'avère pas efficace pour augmenter l'acceptation à des requêtes de don du sang et confirment les recherches antérieures montrant que les techniques d'incitation à l'acceptation ne s'avèrent pas efficientes dans les cas de sollicitation de dons du sang. Cependant, il a été montré que, comparé aux précédentes collectes organisées dans ce même lieu, plus de dons du sang ont été obtenus, attestant que la sollicitation en face à face de donneurs potentiels augmente les dons.

Mots-clés : Contact tactile, acceptation, don du sang

\section{Contexte et apport théoriques}

Après une longue période de diminution des besoins en produits sanguins labiles (plaquettes, plasma...), la situation s’est inversée depuis 2003. Ainsi, les besoins ont évolué de manière constante jusqu’en 2007 sur un rythme de 2 à $3 \%$ par an, soit une augmentation en moins de cinq ans de $10 \%$.

Plusieurs facteurs expliquent cette augmentation des besoins en produits sanguins: le vieillissement de la population; l'impact de la confiance retrouvée dans les produits sanguins après le scandale du sang contaminé ; une plus grande sécurité des produits eux mêmes et des risques transfusionnels qui sont de mieux en mieux maîtrisés; une amélioration constante des techniques médicales et thérapeutiques qui optimise l'utilisation des produits sanguins et enfin les progrès de la médecine qui permettent le traitement de patients par chimiothérapie lourde pour leucémie et/ou cancer à des âges de plus en plus avancés.

On constate depuis quelques années une stagnation du nombre de dons, ce qui est problématique car si le volume de dons ne faiblit pas, il n’augmente pas suffisamment pour couvrir l'ensemble des besoins. Le don moyen annuel par donneur est de 1,6 par an, alors que pour assurer l'autosuffisance en produits sanguins, il faudrait un don moyen annuel égal à deux.

Le propos de cet article, est de rendre compte d'une étude que nous avons menée, sur l'utilisation d'une procédure incitative au don du sang sur de potentiels donneurs. Nous avons testé la technique du toucher afin de faire croître le nombre de donneurs lors d'une collecte de sang organisée sur un site universitaire, par l’Établissement Français du Sang [EFS]. 


\section{L'influence du contact tactile}

Les effets positifs d'un contact tactile sur l'acceptation de sollicitations ont été mis en évidence à de nombreuses reprises dans la littérature psychosociale. Cette méthode simple consiste à assortir une sollicitation quelconque (demande d'aide, demande de direction dans la rue, demande de participation à une enquête...) d'un contact tactile de une à deux secondes la plupart du temps sur l'avant-bras effectué par le requêteur au moment de la formulation verbale de la requête lors d'une interaction en face à face. Dans l'expérience princeps publiée sur le toucher, on a ainsi montré que le fait de toucher quelqu'un dans la rue en formulant une demande de monnaie conduisait les passants à accepter de donner dans des proportions plus élevées qu'en l'absence de contact (Kleinke, 1977). D’autres travaux confirmeront ces résultats initiaux en montrant qu'un contact tactile incite plus favorablement des passants dans le rue à accepter de participer à une enquête sur leur habitudes alimentaires (Hornik, 1987; Hornik \& Ellis, 1988), ou sur les bijoux (Guéguen 2001b, 2002a). Un contact tactile initial s'avère également plus efficace pour inciter des individus à répondre à des questionnaires portant sur des sujets intimes (Nannberg \& Hansen, 1994). Des recherches ont également montré que le comportement du consommateur est influencé par un contact tactile. Ainsi, plusieurs recherches ont confirmé qu'un serveur ou une serveuse touchant un client au moment de la remise du menu ou de la note recevra plus de pourboires qu'en l'absence d'un tel contact (Crusco \& Wetzel, 1984). Le fait de toucher des clients induits de leur part plus d'achat (Smith, Gier \& Willis, 1982; Hornik, 1992a, 1992b; Guéguen, 2001a). Ainsi, l'ensemble de ces recherches semble montrer que le contact tactile a un effet positif sur l'acceptation d'une requête dans le cadre de nombreuses sollicitations. Une méta-analyse a montré que la moyenne de l'importance de l'effet atteignait un bon niveau ( $r$ $=.21$ ) et que celui-ci apparaissait relativement homogène selon les études (Segrin, 1993).

\section{Le toucher dans le cadre médical et de la santé}

Les effets d'un contact tactile sur des comportements en lien avec la santé ont été mis en évidence dans la littérature et cela dans des circonstances impliquant une variété importante de comportements de santé. On a ainsi évalué les effets d'un simple encouragement tactile accompagnant l'aide à la prise alimentaire auprès de patients d'altérations cérébrales chroniques (Eaton, Mitchell-Bonair, \& Friedmann, 1986). Dans le groupe utilisant un encouragement tactile à manger, on observera, comparativement au groupe sans encouragement tactile, une augmentation de la prise calorique de $29 \%$ et de $36 \%$ de la prise de protéines. Les recherches ont également montré qu'un contact tactile est associé à plus de confort et de bien-être 
exprimé par un patient. Des patients touchés par leur psychothérapeute font plus de révélations personnelles que les patients non touchés (Pattison, 1973). On a mis en évidence que des personnes venant pour une première séance de conseil en psychologie clinique sont plus susceptibles d'accepter de poursuivre si elles ont été touchées par le thérapeute (Bacorn \& Dixon, 1984). De la même manière, des patients en gériatrie, lorsqu’ils sont touchés par une infirmière lors d'une interaction, manifestent plus d'interactions sociales que les patients non touchés (Hollinger, 1986). Certaines études montrent également que, dans le cadre psychothérapeutique, le toucher conduit à une perception plus positive du psychothérapeute. Ainsi, on a montré que des patients touchés par leur psychothérapeute jugent par la suite ce dernier comme plus compétent, à l'écoute, empathique et ont une évaluation plus positive de la séance thérapeutique (Alagna, Whitcher, Fisher, \& Wicas, 1979). Ces effets sur le jugement du thérapeute et de la thérapie ont été mis en évidence à plusieurs reprises dans la littérature (Durana, 1998; Hubble, Noble, \& Robinson, 1981; Stockwell \& Dye, 1980). Cependant ces recherches précitées n’ont pas testé l'effet comportemental du toucher. Une étude récente a montré qu’un contact tactile administré par des médecins à leurs patients était associé à une observance plus importante de la prescription médicamenteuse (Guéguen, Meineri, \& Charles-Sire, 2010). Dans leur étude, des médecins avaient pour instruction de très légèrement toucher ou pas le bras de leurs patients en les raccompagnant à l'issue de la consultation. Dans cette étude, il s'agissait de personnes ayant une pharyngite nécessitant la prescription d'un antibiotique. Le contact tactile avait lieu dans le cadre d'une promesse demandée par le médecin au patient de bien suivre la prescription jusqu'au bout. Une semaine après, une mesure de l’observance réelle était effectuée et révélait que les patients préalablement touchés avaient pris plus régulièrement et plus longuement leur prescription que ceux n’ayant pas été touchés.

\section{Augmenter le don du sang}

En considérant ces multiples effets positifs du toucher sur l'acception de différents types de requêtes et notamment celles associés à des comportements de santé ou dans le cadre de consultation de santé, nous avons envisagé de tester l'effet de la technique du contact tactile sur le don du sang. Des recherches antérieures ayant testé l'impact de différentes techniques d'incitation comportementale dans le cadre du don du sang ont montré qu'elles s'avéraient peu efficientes sur ce comportement. Par exemple, la technique dite du Pied-dans-la-porte qui consiste à proposer une petite requête à un individu (par exemple répondre à deux questions sur la santé) puis à lui soumettre une requête plus importante (par exemple accepter une consultation préventive de certains cancers) a eu des effets positifs sur 
des comportements en lien avec la santé et les comportement altruistes sauf pour le don du sang. Avec cette procédure, des chercheurs ne sont pas parvenus, après 3 expériences introduisant des variantes, à augmenter significativement le nombre de personnes non-donneuses acceptant de donner leur sang (Foos \& Dempsey, 1979). De la même manière, la technique de la Porte-dans-le-nez qui consiste à formuler une requête exorbitante qui est refusée puis à proposer une requête d'un coût plus élevée, s'avère également très efficace pour influencer de nombreux comportements altruistes sauf, encore une fois, le don du sang (Cialdini \& Ascani, 1976). Pour les chercheurs, le don du sang implique un tel coût psychologique dans la réalisation de l'acte que ces différentes techniques d'influence ne peuvent briser cet effet négatif initial. Notre objectif a donc été de tester l'effet de la technique du contact tactile sur l'acceptation à donner son sang. En effet, si le don du sang est un comportement suscitant de l'angoisse, on peut comprendre que les techniques de Pied-dans-la-porte ou de Porte-dans-le-nez s'avèrent peu efficaces. Celles-ci supposent en effet des processus cognitifs d'appréciation de la requête et non des effets affectifs. Ainsi, le fait qu'il y ait une première requête conduit à ne pas apprécier le coût de la seconde requête, qui est celle réellement recherchée : cette seconde requête est jugée moins coûteuse qu'en réalité donc conduit à ce qu'elle soit acceptée plus facilement. La technique du contact tactile est relationnelle par nature car toucher, encourager autrui par un contact tactile s'apparente à une marque d'affection et de relations plus intimes entre personnes. Le contact tactile est également utilisé pour apaiser. Dans le cadre du don du sang, il se pourrait ainsi qu'auprès de non-donneurs, lesquels ont l'angoisse de donner lorsque cette demande leur est faite, le contact tactile vient diminuer cette angoisse et, du coup, facilite le passage à l'acte et donc l'acceptation de la requête adressée par le solliciteur. Une évaluation de cette technique a été faite dans le cadre d'une collecte de sang organisée dans un établissement d'enseignement supérieur. Selon le cas, lors de la sollicitation, les étudiants étaient touchés ou pas sur le bras par un volontaire au moment de la formulation de la requête. Nous avons fait l'hypothèse qu'un contact tactile induirait significativement plus d'acceptation de la requête de don.

\section{Méthode}

\section{Participants}

Les participants étaient 281 étudiants de premier cycle en sciences et sciences pour l'ingénieur qui ont été sollicités dans différents endroits d'un bâtiment scientifique sur le campus de l'Université de Bretagne-Sud à Lorient en France. Cent quarante et un étudiants ont été affectés au groupe toucher contre 140 au groupe contrôle sans toucher. 


\section{Procédure}

L'expérience a été menée dans le cadre d'une collecte de sang planifiée sur une demi-journée et réalisée depuis plusieurs années dans cette structure de l'université de Bretagne-Sud. Comme précédemment, une campagne d'annonce par voie d'affichage de la collecte de sang mentionnant le lieu, la date et les horaires a été effectuée à l'intérieur comme à l'extérieur du bâtiment afin d'informer le public. Six étudiants en master Santé Publique (2 hommes et 4 femmes) ont joué le rôle de solliciteurs de dons de sang auprès des étudiants. La sollicitation avait lieu le matin sachant que la phase de collecte de sang proprement dite avait lieu l'après-midi. Chaque compère abordait un étudiant entrant dans le bâtiment de la manière suivante :

«Bonjour, je suis mandaté(e) par l'Etablissement Français du Sang afin de t'informer qu'une collecte de sang est organisée ici cet après-midi salle 8 de 13 h30 à 17h30. Est-ce que tu accepterais de faire un don ? »

On attendait alors la réponse de l'étudiant. Dans le cas d'une réponse positive, on lui remettait alors une carte en prétextant que ce dernier devrait la remettre lorsqu'il/elle irait faire son don. Le solliciteur remerciait alors la personne et lui souhaitait une bonne journée. Selon une méthode de successions aléatoirement déterminée par ordinateur (une suite de A (condition toucher) et $B$ (condition sans toucher) pour chaque personne sollicitée), le solliciteur touchait ou ne touchait pas l'avant-bras de l'étudiant au moment où la prise de contact avait lieu. Afin d'avoir une traçabilité de la condition expérimentale, la carte qui était remise aux étudiants variait selon la condition : une barre verticale se trouvait à droite de la carte pour indiquer une condition tandis qu'elle se trouvait à gauche dans le cas de la seconde condition. Lorsqu'un étudiant se présentait dans la salle dévolue à la collecte, l'employée à l'accueil demandait à l'étudiant si il/elle avait reçue une carte et, si c'était le cas, demandait alors à l'étudiant de la lui remettre.

\section{Résultats et discussion}

Les variables dépendantes mesurées dans cette étude ont été le nombre d'acceptations verbales à donner son sang durant l'interaction entre solliciteur et étudiant (acceptation verbale) et le nombre de participants qui se sont effectivement présentés à la salle de collecte (acceptation comportementale). Les résultats obtenus selon les conditions expérimentales sont présentés dans le tableau 1.

Tableau 1: Fréquences d'acceptation verbale et comportementale selon les conditions expérimentales.

\begin{tabular}{ccc}
\hline & $\begin{array}{c}\text { Toucher } \\
\mathrm{N}=141\end{array}$ & $\begin{array}{c}\text { Pas de toucher } \\
\mathrm{N}=140\end{array}$ \\
\hline Acceptation verbale & $45.4 \%(64 / 141)$ & $47.9 \%(67 / 140)$ \\
\hline Acceptation comportementale & & \\
Par rapport à l'échantillon initial & $11.3 \%(16 / 141)$ & $10.7 \%(15 / 140)$ \\
Par rapport au nombre d'acceptations verbales & $25.0 \%(16 / 64)$ & $22.4 \%(15 / 67)$ \\
\hline
\end{tabular}


Les données ont été analysées à l'aide d’un test d'indépendance. En ce qui concerne l'analyse de l'acceptation verbale de la requête de don, aucune différence entre les conditions expérimentales n'est observée $\left(\chi^{2}(1, N\right.$ $=291)=.17, n s, r=.02$ ). Le contact tactile ne s'avère donc pas efficace pour augmenter significativement le taux de personnes acceptant le principe d'un don. En ce qui concerne le comportement effectif de don, on ne trouve pas non plus de différence significative tant en ce qui concerne les fréquences évaluées par rapport à l'effectif total $\left(\chi^{2}(1, N=291)=.03, n s, r=.01\right)$ que celles par rapport à l'effectif des personnes ayant accepté verbalement de faire un don $\left(\chi^{2}(1, N=131)=.01, n s, r=.03\right)$.

Les résultats obtenus ici ne valident donc pas l'hypothèse d'une augmentation de l'efficacité du toucher sur le taux d'acception d'une requête de don du sang et sur le don effectif. Ces résultats contredisent un nombre important de recherches ayant montré un effet positif du contact tactile sur l'acceptation d'une sollicitation. Plusieurs explications peuvent être avancées à cet échec. Beaucoup de recherches préalables ont montré que le toucher s'avérait efficace dans le cadre de demandes d'aide mais lorsque la sollicitation était au profit du solliciteur. Or, ce n'est pas le cas du don du sang qui ne permet pas d'identifier celui qui en a besoin. Il est ainsi possible que cette technique n'opère que lorsque la sollicitation est essentiellement au profit du solliciteur qui touche en formulant sa demande d'aide. Une seconde explication pourrait résider dans la taille de l'échantillon utilisé ici qui serait insuffisante. Cependant dans les recherches citées précédemment dans la littérature, la plupart des groupes comparés utilisaient des échantillons de 3040 individus (Segrin, 1993; Guéguen \& Joule, 2008) alors que nous avions des effectifs plus importants ici. En outre, aucune variation n'est observée entre les deux conditions. De fait, cette explication liée à la taille de l'échantillon nous semble peu recevable. Une troisième explication peut résider dans la requête de don. Il est possible que le toucher ne soit pas une procédure d'activation comportementale suffisante lorsque la requête porte sur des requêtes au coût psychologique important. Cette absence d'effet d'autres techniques incitatives comme le pied-dans-la-porte ou la porte-dansle-nez a également été mise en évidence dans le cas du don du sang (Foss \& Dempsey, 1979; Cialdini \& Ascani, 1976) alors que ce n'était pas le cas pour des requêtes moins coûteuses psychologiquement pour les individus. Ces recherches ont également été conduites avec des sollicitations de dons effectuées sur des campus ce qui rend la comparaison possible avec le type de population utilisée dans notre propre recherche. Une enquête a ainsi montré que pour les adultes jeunes, donner son sang est associé à la peur de la piqure ou de voir son propre sang (Bazin \& Malet, 2006). Ainsi, un contact tactile de courte durée, non répété est sûrement insuffisant pour diminuer de telles craintes et appréhensions. 
Si ces résultats invalident l'effet d'incitation du toucher en soi, les données de cette campagne éclairent cependant un processus qui montre que, globalement, la méthodologie utilisée ici, peut s’avérer efficace pour augmenter le nombre de dons. En effet, dans le lieu où a été effectuée cette collecte, nous avons pu obtenir les fréquences des dons effectifs recueillis lors des 4 précédentes collectes organisées dans les mêmes lieux les deux années qui ont précédé cette expérimentation. Or, lors de ces collectes antérieures, les étudiants n’étaient pas abordés par des solliciteurs pour donner et étaient simplement informés, classiquement, par voie d’affichage, de l’organisation de la collecte. Les résultats de ces collectes ainsi que ceux obtenus dans le cadre de notre expérimentation sont présentées dans le tableau 2.

Tableau 2: Nombres de dons selon les collectes organisées précédemment

\begin{tabular}{ccc}
\hline & $\begin{array}{c}\text { Nombre de } \\
\text { donneurs }\end{array}$ & $\begin{array}{c}\text { Comparaison dons période } \\
\text { expérimentation et autre période }\end{array}$ \\
\hline Décembre 2009 (experimentation) & 48 & $\chi^{2}(1, N=75)=5.88, p^{*}=.01$ \\
Septembre 2009 & 27 & $\chi^{2}(1, N=79)=3.29, p^{*}=.07$ \\
Janvier 2009 & 31 & $\chi^{2}(1, N=68)=11.50, p^{*}<.001$ \\
Novembre 2008 & 20 & $\chi^{2}(1, N=60)=21.60, p^{*}<.001$ \\
Mai 2008 & 12 &
\end{tabular}

\section{Probabilité unilatérale}

On constate que les dons réels obtenus lors de notre expérimentation apparaissent statistiquement supérieurs à ceux obtenus lors de collectes précédentes réalisées dans les mêmes lieux Nous pouvons donc observer que la communication en face à face conduit à une augmentation des dons puisque l'étude montre que le nombre de donneurs lors de la collecte de décembre 2009 est doublé par rapport à celle de novembre 2008, et quadruplé par rapport à mai 2008. Les préconisations du CerPhi ${ }^{4}$ qui s’est engagé à travailler avec l'EFS sur la communication et le changement de comportement, sont confirmées par cette approche interactionnelle. Le fait d’être sollicité en face à face, par un solliciteur, semble donc une approche qui s'avère efficace pour augmenter significativement le nombre de donneurs. A l'image de l'étude menée par le CREDOC $^{5}$ en $2007^{6}$, 85\% des français citent au moins un frein au don. « Ne pas y penser » ou « ne pas être sollicité » sont les deux raisons les plus souvent citées parmi les freins et, ces raisons, sont encore plus fréquemment évoquées chez les jeunes de 18-24 ans : le groupe d’âge des personnes sollicitées dans notre expérimentation. Donner son sang n'est pas un automatisme, quelque chose auquel on pense

\footnotetext{
${ }^{4}$ Centre d'Etude et de Recherche sur la Philanthropie

${ }^{5}$ Centre de Recherche pour l'Etude et l'Observation des Conditions de vie

${ }^{6}$ CREDOC, Les Français et le don du sang, collection des rapports n²47, octobre 2007
} 
spontanément même si tout le monde reconnaît que c’est un geste indispensable. Ce serait donc aux organismes collecteurs de faire appel au don. Cependant, cette forme de communication interactionnelle demande un investissement humain important, une formation probable des vecteurs des messages, et une logistique adaptée. Beaucoup déclarent manquer de temps pour effectuer ce geste ou ne savent pas où aller pour l'effectuer. On voit ici le poids des contraintes logistiques. Toutefois, ces résultats montrent que la mise en place de sollicitations directes, en face à face, semble s’accompagner d’une efficacité réelle sur le comportement de don.

\section{Conclusion}

Les résultats de cette expérience montrent que l'utilisation d'un contact tactile dans le cadre d'une sollicitation de don du sang ne s'accompagne pas d'une augmentation ni du taux d'acceptation verbale, ni du don effectif. Cela tend à prouver que ce comportement s’avère résistant aux procédures connues et pourtant éprouvées de changement comportemental. Cependant, les résultats montrent que le fait de solliciter directement les personnes en face-à-face augmente significativement le nombre de donneurs réels. Par conséquent, travailler sur des stratégies de sollicitation de ce type en étudiant les facteurs permettant de renforcer leur efficacité serait, semble-t-il, une voie de recherche prometteuse pour augmenter le nombre de donneurs.

\section{References:}

Alagna, Frank, Sheryle,Whitcher, Jeffrey, Fisher, and Edward Wicas. Evaluative reaction to interpersonal touch in a counseling interview. Journal of Counseling Psychology, 26, 465-472, 1979.

Bacorn, Christopher, and David Dixon. The effects of touch on depressed and vocationally undecided clients. Journal of Counseling Psychology, 31, 488-496. 1984.

Bazin, Cécile, and Jacques Malet. Donner son sang en France[Donating blood in France]. Paris, EFS, 2006.

Cialdini, Robert, and Karen Ascani. Test of a concession procedure for inducing verbal, behavioral and further compliance with a request to give blood. Journal of Applied Psychology, 61, 295-300, 1976.

Crusco, April, and Christopher Wetzel. The midas touch: The effects of interpersonal touch on restaurant tipping. Personality and Social Psychology Bulletin, 10, 512-517, 1984.

Durana, Carlos, The use of touch in psychotherapy: Ethical and clinical guidelines. Pychotherapy, 35, 269-280, 1998. 
Eaton, Muzza, Iola, Mitchell-Bonair, and Erika Friedmann. The effect of touch on nutritional intake of chronic organic brain syndrome patients. Journal of Gerontology, 41, 611-616, 1986.

Foss, Robert, and Carolyn, Dempsey. Blood donation and the Foot-in-theDoor technique: A limiting case. Journal of Personality Social Psychology, 37, 580-590, 1979.

Guéguen, Nicolas. Toucher et soumission à une requête : Réplications expérimentales en situation naturelle et évaluation de l'impact du statut [Touch and compliance wiht a request: Experimental replication and status evalaution]. Revue Internationale de Psychologie Sociale, 14, 113-158, 2001a

Guéguen, Nicolas. L'effet d'influence du toucher sur le comportement du consommateur: 2 illustrations expérimentales en extérieur [The effect of touch one consumer behavior : 2 expérimental studies in field setting]. Direction et Gestion: La Revue des Sciences de Gestion, 190-191, 123-132, 2001b.

Guéguen, Nicolas. Touch, awareness of touch and compliance to a request. Perceptual and Motor Skills, 95, 355-360, 2002a.

Guéguen, Nicolas. and Robert-Vincent Joule. L'effet d'influence du toucher sur la soumission à des requêtes: Une méta-analyse [Influence of touch on compliance requests : A meta-analysis]. Cahiers Internationaux de Psychologie Sociale, 80, 39-58, 2008.

Guéguen, Nicolas, Sébastien, Meineri, and Virginie,Charles-Sire. Improving medication adherence by using practitioner nonverbal techniques: A field experiment on the effect of touch. Journal of Behavioral Medicine, 33, 466473, 2010.

Hollinger Linda. Communicating with the elderly. Journal of Gerontology Nursing, 12, 8-13, 1986.

Hornik, Jacob. The effect of touch and gaze upon compliance and interest of interviewees. The Journal of Social Psychology, 127, 681-683, 1987.

Hornik, Jacob. Effects of physical contact on customers' shopping time and behavior. Marketing Letters, 3, 49-55, 1992a.

Hornik, Jacob. Tactile stimulation and consumer response. Journal of Consumer Research, 19, 449-458, 1992b.

Hornik, Jacob, and Shmuel Ellis. Strategies to secure compliance for a mall intercept interview. Public Opinion Quarterly, 52, 539-551, 1988.

Hubble, Mark, Frank, Noble, and Sharon Robinson. The effect of counselor touch in an initial counseling session. Journal of Counseling Psychology, 28, 533-535, 1981.

Kleinke, Chris. Compliance to requests made by gazing and touching experimenters in field settings. Journal of Experimental Social Psychology, 13, 218-223, 1977. 
Nannberg, Jane, and Christine, Hansen. Post-compliance touch: An incentive for task performance. The Journal of Social Psychology, 134, 301-307, 1994. Pattison, Joyce. Effects of touch on self-exploration and the therapeutic relationship. Journal of Consulting and Clinical Psychology, 40, 170-175, 1973.

Segrin, Chris. The effects of nonverbal behavior on outcomes of compliance gaining attempts. Communication Studies, 44, 169-187, 1993.

Smith, David., Joseph, Gier, and Frank Willis. Interpersonal touch and compliance with a marketing request. Basic and Applied Social Psychology, 3, 35-38, 1982.

Stockwell, Samuel, and Allan Dye. Effects of counselor touch on counseling outcome. Journal of Counseling Psychology, 27, 443-446, 1980. 